\title{
MLST Genotypes and Antibiotic Resistance of Campylobacter spp. Isolated from Poultry in Grenada
}

\author{
Diana Stone, ${ }^{1}$ Margaret Davis, ${ }^{2}$ Katherine Baker, ${ }^{2}$ Tom Besser, ${ }^{2}$ \\ Rohini Roopnarine, ${ }^{1}$ and Ravindra Sharma ${ }^{1}$ \\ ${ }^{1}$ School of Veterinary Medicine, St. George's University, P.O. Box 7, Grenada, West Indies \\ ${ }^{2}$ College of Veterinary Medicine, Washington State University, Pullman, WA 99164-7040, USA \\ Correspondence should be addressed to Diana Stone; dstone@sgu.edu
}

Received 7 November 2012; Revised 28 December 2012; Accepted 7 January 2013

Academic Editor: Fabio Ribeiro Braga

Copyright (C) 2013 Diana Stone et al. This is an open access article distributed under the Creative Commons Attribution License, which permits unrestricted use, distribution, and reproduction in any medium, provided the original work is properly cited.

\begin{abstract}
This study determined whether multilocus sequence types (MLST) of Campylobacter from poultry in 2 farms in Grenada, West Indies, differed by farm, antimicrobial resistance and farm antibiotic use. Farm A used fluoroquinolones in the water and Farm $\mathrm{B}$ used tetracyclines. The E-test was used to determine resistance of isolates to seven antibiotics. PCR of the IpxA gene confirmed species and MLST was used to characterize 38 isolates. All isolates were either C. jejuni or C. coli. Farm antibiotic use directly correlated with antimicrobial resistance of Campylobacter isolates. Almost $80 \%$ of the isolates from Farm A were fluoroquinolone resistant and $17.9 \%$ of the isolates from Farm B were fluoroquinolone resistant. All Campylobacter isolates from Farm A were tetracycline sensitive, whereas $35.7 \%$ of isolates from Farm B were tetracycline resistant. Six previously recognized sequence types (STs) and 2 novel STs were identified. Previously recognized STs were those overwhelmingly reported from poultry and humans globally. Isolates with the same ST did not always have the same antibiotic resistance profile. There was little ST overlap between the farms suggesting that within-farm transmission of Campylobacter genotypes may dominate. MLST typing was useful for tracking Campylobacter spp. among poultry units and can help elucidate Campylobacter host-species population structure and its relevance to human health.
\end{abstract}

\section{Introduction}

Since the 1970s, Campylobacter has been recognized as one of the most common causative agents of bacterial foodborne illness $[1,2]$. The genus Campylobacter consists of small Gram-negative, spirally curved rods and it is primarily the thermophilic species of $C$. jejuni and C. coli. that are responsible for human disease [3]. Infection in humans can be asymptomatic or present with a range of clinical symptoms from mild diarrhea to severe diarrhea with fever [4]. Complications may involve reactive arthritis [5] and GuillainBarré syndrome [6]. The incidence of human Campylobacter infections is increasing worldwide, as well as the proportion of isolates resistant to fluoroquinolones, one of the primary classes of drugs used to treat human campylobacteriosis [7]. Animals are the natural reservoir hosts for Campylobacter species but the agent rarely causes disease in the animal host. The most common species of Campylobacter associated with human infections are C. jejuni and C. coli [8]. Both of these species have acquired fluoroquinolone resistance as well as resistance to other antibiotics [9-11]. It has been proposed that the poultry reservoir is the most important source of human infections and, in particular, the source for human fluoroquinolone-resistant Campylobacter isolates [1].

Grenada is a small tri-island state in the Eastern Caribbean. The country's livestock sector consists primarily of poultry, swine, and small ruminant production with poultry products having the highest demand in the local market. In 2009, Grenada produced over 1.5 million lbs of poultry, an increase over previous years. However, over $90 \%$ of meat requirements are met by imported products and an important national goal is to increase local poultry production by $60 \%$ in order to achieve a more self-sufficient market [12].

Information concerning the incidence of human campylobacteriosis in Grenada is not available, but World Health Organization report in 2005 from Grenada stated that 
gastroenteritis was an important cause of morbidity and mortality particularly among infants and children [13, 14]. More recently both $C$. jejuni and $C$. coli have been isolated from poultry in Grenada. Some isolates showed resistance to several antibiotics including fluoroquinolones, and importantly, antibiotic resistance profiles of isolates varied significantly among farms [15-17].

The objective of this study was to determine whether genetic clones of Campylobacter from poultry in Grenada differed by farm, antimicrobial resistance, and farm antibiotic use. To accomplish this multilocus sequence type (MLST), genotyping was performed on all Campylobacter isolates from two broiler farms which differed in their antibiotic use and in their prevalence of antibiotic resistant Campylobacter. MLST genotyping of Campylobacter is an important tool in elucidating the diversity of animal host Campylobacter isolates and provides not only information on transmission routes to humans but also on host-specific emergence of antibiotic resistant clones and their persistence within the animal host and the environment. The results of Campylobacter genetic population structures and antimicrobial resistance patterns within these two broiler farms in Grenada contribute to our understanding of the local and global epidemiology of antibiotic resistant poultry-associated Campylobacter.

\section{Material and Methods}

2.1. Farm Factors. Two poultry farms, designated Farm A and Farm B, participated in the study. A standardized questionnaire was completed at each farm by the owner/manager. Factors evaluated were housing type, flock size, feed composition and source, water source, presence of other domestic animals, use of footbaths, rodent control, husbandry practices, slaughter practices, flock health history, manure spread and disposal methods, and use of antimicrobial drugs [17]. A convenience sample of 25 6-8-week-old broilers were obtained from each farm between September 2009 and May 2011. Samples were collected at the farm immediately after the on-farm slaughter procedure. Cloacal swabs and postevisceration swabs of the abdominal cavity (visceral swabs) were obtained from each bird. Samples were transported on ice to St George's University School of Veterinary Medicine Diagnostic and Research Laboratory for processing in the same day.

2.2. Bacterial Culture and Identification. Cloacal and visceral swabs were plated onto Campylobacter blood-free selective agar with cefoperazone and amphotericin B supplements (modified CCDA Preston plates) (Oxoid Ltd., Basingstoke, UK) and incubated under microaerophilic conditions at $42^{\circ} \mathrm{C}$ for $48 \mathrm{~h}$ using the GENbox microaer system (96126 BioMerieux UK Ltd., Basingstoke, UK). Presumptive Campylobacter colonies were identified by their typical grey-white, mucoid flat appearance on modified Preston CCDA plates. Campylobacter morphology was confirmed by Gram staining. Growths of pure cultures were transferred into $2 \%$ sterile skim milk in cryovials and stored at $-85^{\circ} \mathrm{C}$. To speciate the isolates, the hippurate test (Remel, Lennexa, KS, USA) was used as previously described [15]. Speciation was confirmed on DNA using a multiplex lpxA PCR that discerns between C. jejuni, C. coli, C. lari, and C. upsaliensis, based on amplicon size as previously described [18].

2.3. Antimicrobial Susceptibility Testing. Antimicrobial susceptibility/resistance of Campylobacter isolates was determined for the following seven antibiotics: gentamicin, chloramphenicol, ciprofloxacin, ampicillin, tetracycline, erythromycin, and metronidazole. The E-test (AB Biodisk Solna, Sweden) for minimum inhibitory concentration (MIC) was conducted according to the manufacturer's instructions on Mueller Hinton agar (Remel) as previously described [15]. A C. jejuni strain susceptible to all seven drugs and giving reproducible MICs was used as a control. The MIC of a drug was read directly from the scale printed on the E-test strip at the point of intersection between the zone of bacterial growth and the rest of the strip. Breakpoints established by the Clinical and Laboratory Standards Institute for the microbroth dilution test on aerobic bacteria were used to interpret MICs $[19,20]$. MIC values used to classify a strain as resistant were as follows: ampicillin $\geq 32 \mu \mathrm{g} \mathrm{mL}^{-1}$, chloramphenicol $\geq 32 \mu \mathrm{g} \mathrm{mL}^{-1}$, ciprofloxacin $\geq 4 \mu \mathrm{g} \mathrm{mL}^{-1}$, erythromycin $\geq 8 \mu \mathrm{g} \mathrm{mL}^{-1}$, gentamicin $\geq 16 \mu \mathrm{g} \mathrm{mL}^{-1}$, tetracycline $\geq 16 \mu \mathrm{g} \mathrm{mL}^{-1}$ and metronidazole $\geq 16 \mu \mathrm{g} \mathrm{mL}^{-1}$.

2.4. Multilocus Sequence Typing (MLST). Stock cultures of the 51 isolates were grown on blood agar plates for $24 \mathrm{~h}$ at $42^{\circ} \mathrm{C}$ under microaerophilic conditions. Although most pathogenic species grow at $37^{\circ} \mathrm{C}, \mathrm{C}$. jejuni requires $42^{\circ} \mathrm{C}$ for optimum growth [21]. DNA was extracted using DNeasy Blood \& Tissue Kits (Quagen Inc., Valencia, CA, USA) according to manufacturer's instructions, and samples were shipped to Washington State University College of Veterinary Medicine for MLST determination. Amplification of seven genes was carried out as described previously [22] using primer sequences obtained from http://pubmlst.org/campylobacter/info/primers.shtml. The amplification products were purified by precipitation with $20 \%$ polyethylene glycol $2.5 \mathrm{M}$ $\mathrm{NaCl}$ and sequenced (Functional Biosciences Inc. Madison, WI, USA) using internal sequencing primers described on the same website. Each $20 \mu \mathrm{L}$ amplification reaction mixture contained Custom Genome Services (GCS, Pullman, WA, USA) master mix (14 $\mu \mathrm{L}: 2 \mathrm{U}$ taq polymerase, $0.2 \mathrm{mM}$ dNTP's, $2 \mathrm{mM} \mathrm{Mg}), 1 \mu \mathrm{M}$ each primer, and $2 \mu \mathrm{L}$ gDNA template. PCR cycling conditions were as previously described by Schouls et al. [23], which is a modification of the procedure described by Dingle et al. [22].

The nucleotide sequences of the amplification products were determined at least once on each DNA strand using internal nested primers and BigDye Ready Reaction Mix (PE Biosystems, Foster City, CA, USA) in accordance with the manufacturer's instructions. Unincorporated dye terminators were removed by precipitation of the termination products with $95 \%$ ethanol. Sequence data were submitted to the Campylobacter PubMLST database for allele assignments.

2.5. Data Analysis. A two-tailed Fischer's exact test was used to compare the two farms for the number of broilers samples 
TABLE 1: Clonal complexes and allelic profiles from 38 Campylobacter jejuni/coli strains isolated from broilers in Grenada.

\begin{tabular}{|c|c|c|c|c|c|c|c|c|c|}
\hline \multirow{2}{*}{ Farm } & \multirow{2}{*}{ CC } & \multirow{2}{*}{ ST } & \multicolumn{7}{|c|}{ Allele number } \\
\hline & & & ASP & GLN & GLT & GLY & PGM & TKT & UNC \\
\hline \multirow{6}{*}{ A } & ST-443 & $51(5)$ & 7 & 17 & 2 & 15 & 23 & 3 & 12 \\
\hline & ST- $828^{*}$ & $1173(5)$ & 33 & 39 & 30 & 82 & 113 & 56 & 17 \\
\hline & ST-607 & $2927(1)$ & 166 & 2 & 5 & 10 & 151 & 3 & 1 \\
\hline & ST-353 & $353(3)$ & 7 & 17 & 5 & 2 & 10 & 3 & 6 \\
\hline & & $5490(2)^{\dagger}$ & 7 & 84 & 5 & 2 & 11 & 1 & 6 \\
\hline & $\neq$ & $5491(4)^{\dagger}$ & 129 & 39 & 65 & 82 & 113 & 47 & 17 \\
\hline \multirow{3}{*}{ B } & ST-354 & $354(13)$ & 8 & 10 & 2 & 2 & 11 & 12 & 6 \\
\hline & ST-464 & $464(3)$ & 24 & 2 & 2 & 2 & 10 & 3 & 1 \\
\hline & $\ddagger$ & $5491(2)^{\dagger}$ & 129 & 39 & 65 & 82 & 113 & 47 & 17 \\
\hline
\end{tabular}

Numbers in parentheses after each ST denote the number of isolates.

CC: clonal complex.

ST: sequence types.

* Clonal complex associated with C. coli.

${ }^{\dagger}$ Novel STs identified.

${ }^{\ddagger}$ No clonal complex assigned yet.

that were positive for Campylobacter, the number of C. coli and C. jejuni isolates, and the percent of isolates from each farm resistant to each of the seven antibiotics tested. A value of $P \leq 0.05$ was considered significant. Sequence analysis and determination of ST and clonal complex were performed with the online-based MLST application for Campylobacter provided by SmartGene (Zug, Switzerland), which uses the PubMLST database http://pubmlst.org/campylobacter/ for ST designation.

A neighbor-joining tree based on allelic sequences was constructed using the PHYLIP suite of programs available on the PubMLST web site http://pubmlst.org/analysis/. Tree drawing was performed using Phylodendron software also available at the same PubMLST site. Tree branches were labeled with corresponding clonal complex assignments.

\section{Results}

3.1. Farm Information. Both farms were located in the parish of St. Patrick and were separated by about two miles. At the time of sampling, Farm A housed approximately 8000 broilers and Farm B housed approximately 1000 broilers and 3000 layers. Only broilers were sampled for this study. Chickens from Farm A received the fluoroquinolone antibiotic, Norfloxacin, in their water. Chickens from Farm B received the tetracycline antibiotic, Oxytetracycline, in their water. Other than farm size, the type of antibiotic provided in the water and the fact that Farm B raised both broilers and layers, these farms did not differ in their management practices as determined by visual inspection of the premises and owner/manager responses to a questionnaire [17].

3.2. MLST and Campylobacter Strain Results. A total of 51 Campylobacter spp. isolates were obtained from broiler chickens on two farms in Grenada, Farm A (23 isolates; 20 from cloacal swabs and three from visceral swabs) and Farm B (28 isolates; 27 from cloacal swabs and one from a visceral swab). Based on this sample size, the farms did not differ significantly on the percent of sampled broilers positive for Campylobacter (88\% Farm A and 96\% Farm B, P = 0.6092) or on the percent of isolates that were identified as C. coli and C. jejuni by hippurate hydrolysis (8 C. coli and 15 C. jejuni isolates from Farm A and 13 C. coli and 15 C. jejuni isolates from Farm B, $P=0.5681$ ).

MLST genotyping was completed on 38 of the 51 isolates. For the other 13 isolates, ST determination was not possible because amplification products were not detected for one or more loci. These isolates represented both C. jejuni and C. coli. Six clonal complexes identified in this study have been previously described (STs-443, -828, -607, -353, -354, and -464). In addition, two novel STs were detected. One of the novel isolates, ST-5490, was assigned to the 353 clonal complex. The other novel isolate, ST-5491, has not yet been assigned to a clonal complex. One of the clonal complexes, ST-353, included two different STs, ST-353 and the novel ST-5490. Isolates from Farm A consisted of four previously reported STs $(443,353,607$, and 828) and two novel STs, ST- 5490 within the 353 clonal complex (two isolates) and ST-5491 (four isolates). Isolates from Farm B consisted of two PubMLST-reported STs (ST-354 and ST-464) and one novel ST, the ST-5491 (two isolates). This novel 5491 ST was the only MLST ST shared between the two farms. The two novel STs accounted for eight (21\%) of the 38 typable isolates. These STs have been submitted to the PubMLST data base. Overall, the predominant ST was the ST-354 complex with 13 isolates (35\%) and was only observed in Campylobacter isolates from Farm B (Table 1). The six previously reported clonal complexes detected in this study are associated with infections in chickens and humans (Table 2) and five of these are reported globally (Table 3 ).

All isolates with confirmed STs were phenotypically typed by the hippurate test as either C. jejuni or C. coli, with 21 of the 38 isolates (55.3\%) identified as C. jejuni and $11(28.9 \%)$ of the isolates identified as C. coli. Among the C. jejuni and C. coli isolates typed in this study, six clonal complexes were identified for each, four of which included isolates of both 
TABLE 2: The distribution of source host species for clonal complexes detected in Grenada Campylobacter jejuni/colibroiler isolates represented in the international PubMLST database.

\begin{tabular}{|c|c|c|c|c|c|c|}
\hline Source ${ }^{*}$ & $\begin{array}{l}\text { ST-353 clonal } \\
\text { complex } \\
N(\%)\end{array}$ & $\begin{array}{l}\text { ST-354 clonal } \\
\text { complex } \\
N(\%)\end{array}$ & $\begin{array}{c}\text { ST-443 clonal } \\
\text { complex } \\
N(\%)\end{array}$ & $\begin{array}{l}\text { ST-464 clonal } \\
\text { complex } \\
N(\%)\end{array}$ & $\begin{array}{c}\text { ST-607 clonal } \\
\text { complex } \\
N(\%)\end{array}$ & $\begin{array}{c}\text { ST-828 clonal } \\
\text { complex } \\
N(\%)\end{array}$ \\
\hline Ruminant & $2(0.5)$ & $10(4.2)$ & $2(0.9)$ & $0(0)$ & $1(0.8)$ & $162(10.4)$ \\
\hline Chicken & $92(22.8)$ & $64(27.1)$ & $60(27.4)$ & $24(24)$ & $35(29.7)$ & $523(33.4)$ \\
\hline Other poultry & 7 (1.7) & $3(1.3)$ & $1(0.5)$ & $1(1)$ & $0(0)$ & $96(6.1)$ \\
\hline Human & $301(74.7)$ & $158(66.9)$ & $156(71.2)$ & $75(75)$ & $82(69.5)$ & $378(24.2)$ \\
\hline Pig & $1(0.2)$ & $1(0.4)$ & $0(0)$ & $0(0)$ & $0(0)$ & 405 (25.9) \\
\hline Total & $403(100.0)$ & $236(100.0)$ & $219(100.0)$ & $100(100.0)$ & $118(100.0)$ & $1564(100.0)$ \\
\hline
\end{tabular}

${ }^{*}$ Ruminant: calf, cattle, cow's milk, goat, sheep; Chicken: chicken, chicken offal or meat; Other poultry: goose, turkey; Human: human stool, human blood, human unspecified; Pig: pig, pork offal or meat.

species. Two of the clonal complexes included only C. jejuni isolates and two of the clonal complexes were observed only among $C$. coli isolates. The most frequent $C$. jejuni clonal complex was ST-354 (strain type 354) containing 11 of the 21 C. jejuni isolates. The seven C. jejuni STs identified in this study account for $19.4 \%$ of the C. jejuni human stool isolates since 2005 [24]. One of the two C. coli STs was the previously reported ST-828-1173 which contained five of the 11 C. coli isolates. This ST accounted for $74.6 \%$ of the C. coli human stool isolates reported to PubMLST between 2005 and 2011. Six of the 11 C. coli isolates were identified as the novel ST5491, which was the only ST of this species observed on both farms.

A cladogram constructed from the 38 Campylobacter MLST allelic profiles illustrates the clustering of the STs identified in this study. Some isolates from different farms occurred on branches that were closer than isolates within the same farm. For example, ST-464 clonal complex isolates from Farm B occupy the same branch in the tree as the ST607 isolate from Farm A. Two clonal complex ST-353 isolates from Farm A (ST 5490) also branched off the same branch as clonal complex ST-354 isolates from Farm B (Figure 1).

3.3. Antibiotic Resistance. Campylobacter isolates from Farms $\mathrm{A}$ and $\mathrm{B}$ were significantly different with regard to resistance to gentamicin, ciprofloxacin, tetracycline, and metronidazole (Table 4). Seventeen of the 23 Campylobacter isolates from Farm A (73.9\%) were resistant to ciprofloxacin whereas only five of the 28 isolates from Farm B (17.9\%) were resistant to this antibiotic. None of the Campylobacter isolates from Farm A were resistant to tetracycline whereas 10 of the 28 isolates from Farm B (35.7\%) were resistant to this class of antibiotic. Isolates from Farm B showed resistance to more classes of antibiotics and higher rates of resistance than isolates from Farm A. The farms did not differ significantly in the percent of isolates with multidrug resistance (30\% of isolates from Farm A; 35.7\% from Farm B). An analysis of all 51 Campylobacter isolates from these two farms showed that there was no significant difference between the percent of $C$. jejuni isolates resistant to at least one of the tested antibiotics (83\%) and the percent of antibiotic resistant C. coli isolates (81\%). Further, there was no significant difference between $C$. jejuni and
C. coli isolates for the percent of resistance to ciprofloxacin ( $47 \%$ and $43 \%$, resp.) or tetracycline (17\% and $24 \%$, resp.) (Fischer's exact two-tailed test, $P>0.05$ ).

All fluoroquinolone resistant isolates, regardless of MLST type, were from Farm A. All tetracycline resistant isolates, regardless of MLST type, were from Farm B. Multiple isolates of seven of the eight STs were obtained in this study. Isolates from only one ST for which multiple isolates were obtained (ST-353) showed a consistent antibiotic sensitivity/resistance profile, with all three isolates originating from Farm A and being resistant to fluoroquinolone and susceptible to tetracycline. Within each of the STs representing the remaining isolates, there was no consistent antibiotic resistance phenotype for fluoroquinolone or tetracycline. On Farm A, two of the four ST-443 isolates, four of the five ST-828 isolates, and one of the two ST-5490 isolates were resistant to fluoroquinolone whereas the others were sensitive. On Farm B, three of the 13 ST-354 isolates and one of the two ST-464 isolates were resistant to tetracycline whereas the others were sensitive. On Farm B, both of the novel 5491 isolates were sensitive to fluoroquinolone, but four of the isolates from Farm A were resistant to that antibiotic (Table 5).

In one bird from Farm A, Campylobacter spp. were isolated from both the visceral and cloacal swabs for which complete MLST allelic profiles were obtained. The visceral swab isolate was assigned to ST-828 and was phenotypically identified as $C$. coli, whereas the cloacal swab isolate typed to ST-353 and was C. jejuni. Both isolates were resistant to fluoroquinolone and susceptible to tetracycline. Three of the visceral swab isolates from Farm B were typed to ST-354 and all were $C$. jejuni. Two of these isolates were obtained from broilers with MLST typable cloacal swab isolates. The ST of one of the cloacal swab isolates matched the ST of the visceral swab isolate from the same bird, ST-354. The visceral swab isolate was susceptible to tetracycline, whereas the cloacal swab isolate from this bird was resistant to tetracycline. Both isolates were susceptible to fluoroquinolone. The visceral and cloacal swab isolates from the other Farm B bird were both C. jejuni but did not match on either their MLST ST designation or their antibiotic susceptibility/resistance profiles. The MLST of the cloacal swab isolate was ST-464 and was resistant to tetracycline, whereas the MLST of the visceral 
TABLE 3: Campylobacter clonal complex STs identified in broilers from farms A and B that have been previously reported to PubMLST from Grenada and other countries.

\begin{tabular}{lccccc}
\hline & ST-353 & ST-354 & ST-443 & ST-464 & ST-607 \\
2927 & ST-828 \\
1173
\end{tabular}

TABle 4: Antibiotic sensitivity/resistance profiles of 51 Campylobacter jejuni/coli isolates from broilers on Farms A and B in Grenada.

\begin{tabular}{lccc}
\hline Antibiotic $^{*}$ & $\begin{array}{c}\text { Farm A } \\
\text { Sen/Resist (\%R) }\end{array}$ & $\begin{array}{c}\text { Farm B } \\
\text { Sen/Resist (\%R) }\end{array}$ & $\begin{array}{c}P \text { value comparing } \\
\% \text { resistant }\end{array}$ \\
\hline Gen & $23 / 0(0 \%)$ & $22 / 6(21.4 \%)$ & $\boldsymbol{P}=\mathbf{0 . 0 2 6 5}$ \\
Chlor & $22 / 1(0.04 \%)$ & $25 / 3(10.7 \%)$ & $P=0.6173$ \\
Cipro $^{\ddagger}$ & $6 / 17(73.9 \%)$ & $23 / 5(17.9 \%)$ & $\boldsymbol{P}=\mathbf{0 . 0 0 0 1}$ \\
Amp & $20 / 3(13 \%)$ & $27 / 1(3.6 \%)$ & $P=0.3158$ \\
Tetra & $23 / 0(0 \%)$ & $18 / 10(35.7 \%)$ & $\boldsymbol{P}=\mathbf{0 . 0 0 1 1}$ \\
Eryth & $23 / 0(0 \%)$ & $23 / 5(17.9)$ & $P=0.0562$ \\
Metroni & $17 / 6(26.1 \%)$ & $7 / 21(75.0 \%)$ & $\boldsymbol{P}=\mathbf{0 . 0 0 0 7}$ \\
\hline
\end{tabular}

${ }^{*}$ Gen: gentamicin; Chlor: chloramphenicol; Cipro: ciprofloxacin; Amp: ampicillin; Tetra: tetracycline; Eryth: erythromycin; Metroni: metronidazole.

${ }^{\dagger}$ Fischer's exact two-tailed test.

${ }^{\ddagger}$ Antibiotic used on Farm A.

\$Antibiotic used on Farm B.

swab isolate was ST-354 and was susceptible to tetracycline. Both isolates were susceptible to fluoroquinolone.

\section{Discussion}

The aims of this study were to determine the prevalence, genetic diversity, and antibiotic resistance of Campylobacter isolates from broilers at slaughter on two farms in Grenada that differed in antimicrobial use. The high Campylobacter prevalence detected in broilers in this study is in agreement with previous findings in Grenada [15], Switzerland [25], France [26], Senegal [27], and Canada [28]. Also similar to those reports, the majority of isolates from this study were C. jejuni. In the earlier Grenada study, however, C. coli accounted for the majority of the isolates $(60.9 \%$ of isolates compared to $32.8 \%$ for $C$. jejuni) which differed significantly with the results of the current study $(P=0.0154)$. The previous Grenada study [15] also identified C. lari isolates whereas it was not identified in broilers in this study. These differing results may reflect sampling time-line differences, different farms, farm facility or management differences, or sampling biases. Regardless of this, both C. jejuni and C. coli are prevalent in broilers in Grenada.

In this study, eight STs were identified within six previously reported clonal complexes. Two of the STs identified were novel genotypes. One of these, ST-5491, was the only ST identified on both farms and may represent a local clone. The lack of farm MLST overlap suggests that farmspecific transmission pathways may predominate. However, a larger sample size at additional time points will be needed to assess this possibility. If farm-specific transmission of Campylobacter spp. does characterize Grenada poultry operations, it may reflect the current vertically integrated nature of poultry production where each farm controls all stages of production from day-old chicks through slaughter and processing. However, the cluster analysis (Figure 1) indicates 
TABLE 5: Fluoroquinolone and tetracycline sensitivity/resistance of Campylobacter jejuni/coli isolates by MLST profile from broilers on Farms $A$ and $B$ in Grenada.

\begin{tabular}{|c|c|c|c|c|}
\hline \multirow{2}{*}{ Farm/antimicrobial used } & \multirow{2}{*}{$\mathrm{CC}$} & \multirow{2}{*}{ ST } & \multicolumn{2}{|c|}{ Antimicrobial sensitivity/resistance } \\
\hline & & & Fluoroquinolone & Tetracycline \\
\hline \multirow{9}{*}{ Farm A-fluoroquinolone } & ST-443 & $51(3)$ & $\mathrm{S}$ & S \\
\hline & & $51(2)$ & $\mathbf{R}$ & $\mathrm{S}$ \\
\hline & ST-828 & $1173(1)$ & S & S \\
\hline & & $1173(4)$ & $\mathbf{R}$ & S \\
\hline & ST-607 & $2927(1)$ & $\mathbf{R}$ & $\mathrm{S}$ \\
\hline & ST-353 & $353(3)$ & $\mathbf{R}$ & S \\
\hline & & $5490(1)$ & S & S \\
\hline & & $5490(1)$ & $\mathbf{R}$ & S \\
\hline & No CC assigned & $5491(4)$ & $\mathbf{R}$ & $\mathrm{S}$ \\
\hline \multirow{5}{*}{ Farm B-tetracycline } & ST-354 & $354(10)$ & $\mathrm{S}$ & $\mathrm{S}$ \\
\hline & & $354(3)$ & S & $\mathbf{R}$ \\
\hline & ST-464 & $464(2)$ & S & $S$ \\
\hline & & $464(1)$ & S & $\mathbf{R}$ \\
\hline & No CC assigned & $5491(2)$ & S & S \\
\hline
\end{tabular}

CC: clonal complex.

ST: sequence types.

Numbers in parentheses after each ST denote the number of isolates.

S: sensitive.

$\mathrm{R}$ : resistant.

that closely related STs were found on both farms suggesting possible interfarm transmission at some point.

Results of the MLST analysis suggest limited Campylobacter population diversity on Farm B with three STs and ST-354 accounting for over $72 \%$ of all isolates. In contrast, isolates from Farm A reflected six STs and no single ST accounted for more than $25 \%$ of the isolates. Predominant STs at a particular farm have been reported in several studies [29-31], and experimentally some strains are able to become dominant within a confined host-species population [32]. One study identified age of flock broilers as the most important factor correlated with increasing genetic diversity of Campylobacter, implying that genetic clones can accumulate within the flock and persist [33]. It is unlikely that the greater MLST diversity on Farm A compared to Farm B observed in this study reflects any difference in age of flock broilers or length of time these farms have been in production. Both of these farms were reestablished several months after being destroyed by Hurricane Ivan in 2004 and thus have been in continuous production for the same amount of time. Other factors that might contribute to differences in MLST diversity, such as antibiotic use and biosecurity measures prior to our study, or current proximity to other animal agriculture are unknown. A larger isolate set, however, might reveal a more diverse Campylobacter population on Farm B than what was identified in this study.

The major lineages identified in this study correspond to clonal complexes that are primarily associated with poultry and human disease (Table 2) and, except for ST-607, are geographically widely distributed (Table 3 ). Four of the six clonal complexes have been previously reported from Grenada $[16$, 24]. In general, results from this study support the previous observation that host association of Campylobacter genotypes transcends geographic variation [34]. It is of interest that Grenada is a tourist and medical/veterinary educational destination for people from the UK, the European community, and the US. This movement of humans and their pets to the island may impact the island's Campylobacter genotypic population structure.

In the study reported here, $C$. coli isolates represented a more restricted ST population structure than the C. jejuni isolates which is in agreement with a report from the UK [34]. Only one previously recognized C. coli clonal complexST was identified (ST-828-1173, and one novel ST (5491)). Of interest is that the previously reported C. coli ST-828 has been reported in several different countries and host species (Tables 2 and 3) and accounts for $74.6 \%$ of the C. coli human isolates reported to PubMLST. In a US study, C. coli isolates from both human cases and retail meats (beef, pork, and poultry) tended to cluster in the ST-828 complex with the majority of isolates showing ciprofloxacin, erythromycin, or multidrug resistance [35].

The fluoroquinolone and tetracycline resistance profiles of the 51 Campylobacter isolates showed a marked difference between Farm A (high ciprofloxacin resistance) and Farm B (high tetracycline resistance) that directly correlated with farm use of these antimicrobial classes. These results are compatible with antibiotic pressure contributing to both fluoroquinolone and tetracycline resistance, an association that has previously been described for both of these antibiotics [26, 36-39]. On Farm B, greater than 35\% of the Campylobacter isolates were tetracycline resistant which is in agreement with several other studies on broilers [26, 28, 40-42]. Both C. jejuni and C. coli isolates from Farm A were resistant to ciprofloxacin, in agreement with other reports [26, 27, 41]. The percent of fluoroquinolone-resistant isolates on Farm A 


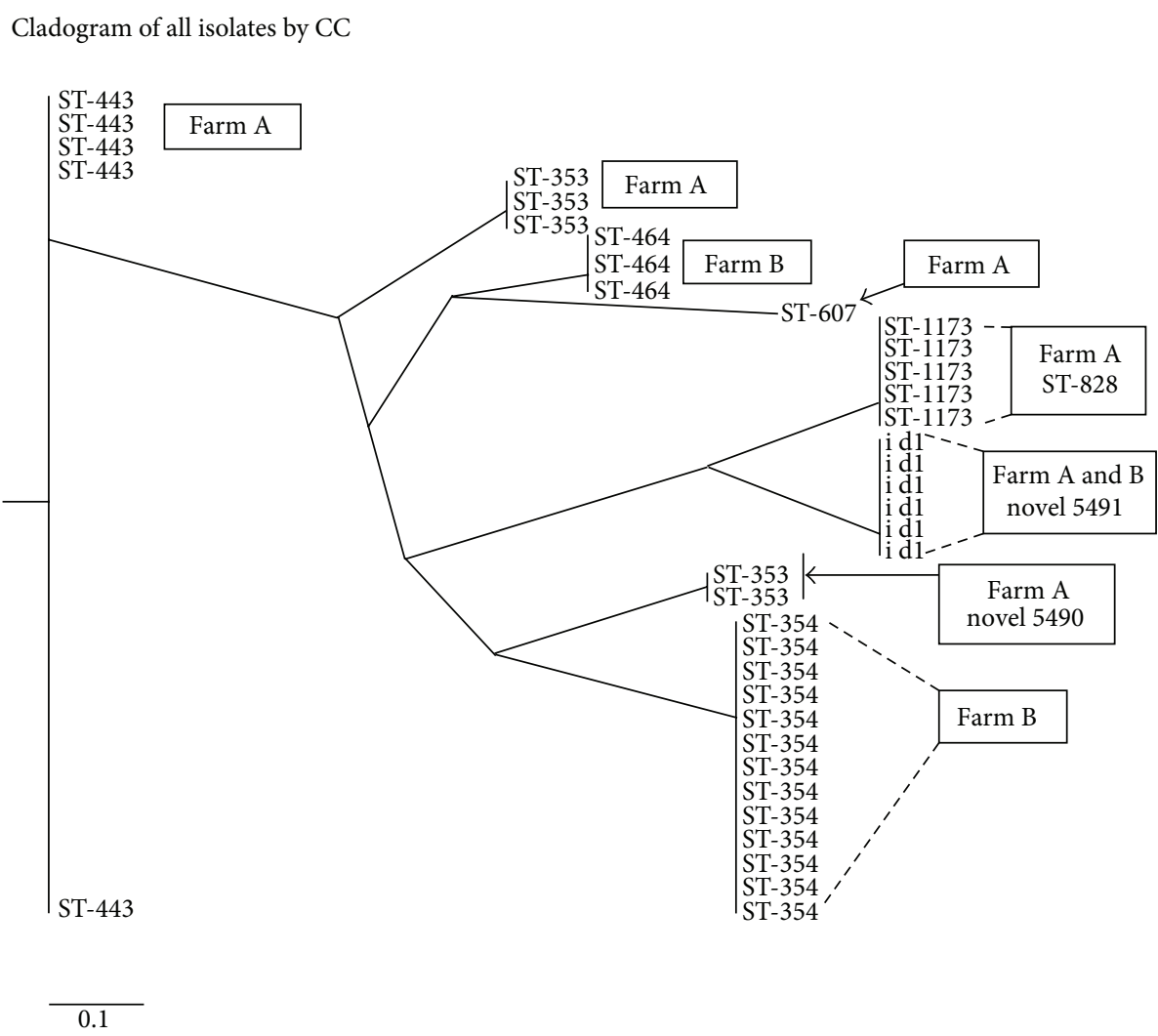

FIGURE 1: Neighbor-joining tree constructed from eight STs identified among 38 Campylobacter jejuni/coli isolates from broilers in Grenada at slaughter on two farms (A and B). Tree branches are labeled with the corresponding clonal complex assignments.

was high (73.9\%), in agreement with other reports from farms where fluoroquinolones were used [41-45], and much higher than reports from countries where fluoroquinolones are banned or use is controlled [46]. Fluoroquinolone resistance on Farm A was also significantly higher than the $12.5 \%$ previously reported for poultry isolates from Grenada [15]. This difference may be due to the fact that data from the previous study included isolates from 10 farms and it is unlikely that fluoroquinolones were used on all farms. Isolates from the two farms in the present study also differed significantly for resistance to gentamicin and metronidazole, neither of which were used on these farms. Importantly, it is also recognized that fluoroquinolone-resistant Campylobacter spp. have been detected on farms that never used that class of antibiotic $[47,48]$. Thus, factors in addition to farm use of antibiotics are likely contributing to antibiotic-resistant Campylobacter spp. in broilers in Grenada.

Although antibiotic use did correlate with fluoroquinolone and tetracycline resistance, within each farm there was no association between MLST genotype and antibiotic resistance. This is similar to other reports for fluoroquinolone resistance $[49,50]$. However, some studies report an association between specific MLST genotypes and resistance to fluoroquinolones $[25,51,52]$, tetracycline $[51,52]$ or multidrug resistance which includes fluoroquinolones and tetracycline [53]. Where evaluated, STs associated with fluoroquinolone resistance contained mutations in the gyrA gene that is responsible for quinolone resistance, suggesting that either specific Campylobacter genotypes are prone to gyrA mutations or that clonal dissemination of resistant STs has occurred or that only a limited range of gyrA mutations confer fluoroquinolone resistance. Although gyrA mutations were not confirmed in the six STs on Farm A with fluoroquinolone resistance, results indicate that mutations in this gene may occur in a number of different STs on a single farm.

Although further studies will be needed to determine a statistical association between antibiotic use and resistant Campylobacter strains, the presence of fluoroquinoloneand tetracycline-resistant Campylobacter isolates concurrent with farm use of these antibiotics in poultry production in Grenada indicates that there may be a need for more judicial use of antimicrobials on the island. Grenada is a small island state far from the US, UK, Europe, and Asia, and resistant Campylobacter strain emergence in Grenada could contribute to resistance globally. It has been noted that foreign travel is a risk factor for fluoroquinolone-resistant campylobacteriosis [54-56]. Furthermore, in popular European travel destinations, both fluoroquinolone and tetracycline resistance among poultry isolates is higher than in other locations [57]. Thus, the movement of people may play a role in transporting antibiotic-resistant strains to other locations. Tourism is the main industry in Grenada and protecting that economic resource, along with resident human health, is critical for this island state. 
In Grenada, poultry production is a vertically integrated, open air system of relatively small production units. Poultry production is often in close association with back yard or small-scale sheep, goat, and swine production units. Thus, there is ample opportunity for movement of Campylobacter spp. among these host species. This type of animal agriculture is common in many parts of the world and findings from Grenada may shed light on Campylobacter population structure, spread, and resistance relevant to many geographic areas. Data from studies of Campylobacter in sheep, goats, and pigs across all parishes in Grenada show low shedding rates in sheep and goats (3-4\%, unpublished data) and high rates in pigs (72\%) [58]. Antimicrobial and MLST analysis will determine whether any of these mammalian isolates correspond to isolates identified in poultry. This, along with genetic and antimicrobial resistance analysis of human case isolates, will give a more comprehensive picture of Campylobacter host-species population structure and its relevance to human health in Grenada and to campylobacteriosis in countries with similar food animal production systems.

\section{Conflict of Interests}

None of the authors have any conflict of interests relating to this study.

\section{Acknowledgments}

This work was funded by a grant from St. George's University Small Research Grant Initiative. This publication made use of the C. jejuni MLST website (http://pubmlst.org/campylobacter/) developed by Keith Jolley and Man-Suen Chan and sited at the University of Oxford [59]. The development of this site has been funded by the Wellcome Trust.

\section{References}

[1] K. Smith, Epidemiology of Campylobacter in Humans, Food and Drug Administration, US Department of Health \& Human Services, 2009.

[2] http://www.cdc.gov/nczved/divisions/dfbmd/diseases/campylobacter/.

[3] J. E. Moore, D. Corcoran, J. S. G. Dooley et al., "Campylobacter," Veterinary Research, vol. 36, pp. 351-382, 2005.

[4] J. M. Ketley, "Pathogeneis of enteric infection by Campylobacter," Microbiology, vol. 143, no. 1, pp. 5-21, 1997.

[5] R. A. Hughes and A. C. Keat, "Reiter's syndrome and reactive arthritis: a current view," Seminars in Arthritis and Rheumatism, vol. 24, no. 3, pp. 190-210, 1994.

[6] I. Nachamkin, B. M. Allow, and T. Ho, "Campylobacter species and Guillain-Barré syndrome," Clinical Microbiology Reviews, vol. 11, pp. 555-567, 1998.

[7] J. E. Moore, M. D. Barton, I. S. Blair et al., "The epidemiology of antibiotic resistance in Campylobacter," Microbes and Infection, vol. 8, no. 7, pp. 1955-1966, 2006.

[8] S. M. Horrocks, R. C. Anderson, D. J. Nisbet, and S. C. Ricke, "Incidence and ecology of Campylobacter jejuni and coli in animals," Anaerobe, vol. 15, no. 1-2, pp. 18-25, 2009.

[9] M. K. Lee, S. J. Billington, and L. A. Joens, "Potential virulence and antimicrobial susceptibility of Campylobacter jejuni isolates from food and companion animals," Foodborne Pathogens and Disease, vol. 1, no. 4, pp. 223-230, 2004.

[10] E. Rahimi, "Occurrence and resistance to antibiotics of Campylobacter spp. in retail raw sheep and goat meat in Shahr-e Kord, Iran," Global Veterinary, vol. 4, pp. 504-509, 2010.

[11] E. Rahimi, M. Ameri, and H. R. Kazemeini, "Prevalence and antimicrobial resistance of Campylobacter species isolated from raw camel, beef, lamb, and goat meat in Iran," Foodborne Pathogens and Disease, vol. 7, no. 4, pp. 443-447, 2010.

[12] Anonymous, Grenada Ministry of Agriculture, Annual Agriculture Review publication.

[13] http://www.paho.org/hia/archivosvol2/paisesing/Grenada\%20 English.pdf\#search="gast roenteritis Grenada.

[14] http://new.paho.org/carec/dmdocuments/9.\%20GE.pdf.

[15] H. Hariharan, S. Sharma, A. Chikweto, V. Matthew, and C. DeAllie, "Antimicrobial drug resistance as determined by the E-test in Campylobacter jejuni, C. coli, and C. lari isolates from the ceca of broiler and layer chickens in Grenada," Comparative Immunology, Microbiology and Infectious Diseases, vol. 32, no. 1, pp. 21-28, 2009.

[16] R. S. Miller, W. G. Miller, M. Behringer, H. Hariharan, V. Matthew, and O. A. Oyarzabal, "DNA identification and characterization of Campylobacter jejuni and Campylobacter coli isolated from caecal samples of chickens in Grenada," Journal of Applied Microbiology, vol. 108, no. 3, pp. 1041-1049, 2010.

[17] R. Roopnarine, D. Stone, H. Hariharan et al., "Fluoroquinolone and metronidazole resistance of Campylobacter spp from broiler chickens and antimicrobial use on farms in Grenada, West Indies," Journal of Animal Research, vol. 2, no. 3, pp. 219-227, 2012.

[18] A. M. Werno, J. D. Klena, G. M. Shaw, and D. R. Murdoch, "Fatal case of Campylobacter lari prosthetic joint infection and bacteremia in an immunocompetent patient," Journal of Clinical Microbiology, vol. 40, no. 3, pp. 1053-1055, 2002.

[19] P. Luber, E. Bartelt, E. Genschow, J. Wagner, and H. Hahn, "Comparison of broth microdilution, E test, and agar dilution methods for antibiotic susceptibility testing of Campylobacter jejuni and Campylobacter coli," Journal of Clinical Microbiology, vol. 41, no. 3, pp. 1062-1068, 2003.

[20] E. Guévremont, É. Nadeau, M. Sirois, and S. Quessy, “Antimicrobial susceptibilities of thermophilic Campylobacter from humans, swine, and chicken broilers," Canadian Journal of Veterinary Research, vol. 70, no. 2, pp. 81-86, 2006.

[21] P. U. Quinn, B. K. Markey, F. C. Leonard, E. S. FitzPatrick, S. Fanning, and P. J. Hartigen, "Campylobacter and Helicobacter species," in Veterinary Microbiology and Microbial Disease, p. 345, Blackwell, West Sussez, UK, 2nd edition, 2011.

[22] K. E. Dingle, F. M. Colles, R. Ure et al., "Molecular characterization of Campylobacter jejuni clones: a basis for epidemiologic investigation," Emerging Infectious Diseases, vol. 8, no. 9, pp. 949-955, 2002.

[23] L. M. Schouls, S. Reulen, B. Duim et al., "Comparative genotyping of Campylobacter jejuni by amplified fragment length polymorphism, multilocus sequence typing, and short repeat sequencing: strain diversity, host range, and recombination," Journal of Clinical Microbiology, vol. 41, no. 1, pp. 15-26, 2003.

[24] http://pubmlst.org/analysis.

[25] S. E. Wirz, G. Overesch, P. Kuhnert, and B. M. Korczak, "Genotype and antibiotic resistance analyses of campylobacter isolates from ceca and carcasses of slaughtered broiler flocks," Applied and Environmental Microbiology, vol. 76, no. 19, pp. 6377-6386, 2010. 
[26] L. Avrain, F. Humbert, R. L’Hospitalier, P. Sanders, C. VernozyRozand, and I. Kempf, "Antimicrobial resistance in Campylobacter from broilers: association with production type and antimicrobial use," Veterinary Microbiology, vol. 96, no. 3, pp. 267-276, 2003.

[27] E. Cardinale, J. A. Dromigny, F. Tall, M. Ndiaye, M. Konte, and J. D. Perrier Gros-Claude, "Antimicrobial susceptibility of Campylobacter strains isolated from chicken carcasses in Senegal," Revue d'Elevage et de Medecine Veterinaire des Pays Tropicaux, vol. 55, pp. 259-264, 2002.

[28] A. Deckert, A. Valdivieso-Garcia, R. Reid-Smith et al., "Prevalence and antimicrobial resistance in Campylobacter spp. Isolated from retail chicken in two health units in Ontario," Journal of Food Protection, vol. 73, no. 7, pp. 1317-1324, 2010.

[29] W. F. Jacobs-Reitsma, C. A. Kan, and N. M. Bolder, "The induction of quinolone resistance in Campylobacter bacteria in broilers by quinolone treatment," Letters in Applied Microbiology, vol. 19, no. 4, pp. 228-231, 1994.

[30] E. Cardinale, V. Rose, J. D. Perrier Gros-Claude et al., "Genetic characterization and antibiotic resistance of Campylobacter spp. isolated from poultry and humans in Senegal," Journal of Applied Microbiology, vol. 100, no. 1, pp. 209-217, 2006.

[31] T. Alter, R. M. Weber, A. Hamedy, and G. Glünder, "Carryover of thermophilic Campylobacter spp. between sequential and adjacent poultry flocks," Veterinary Microbiology, vol. 147, no. 1-2, pp. 90-95, 2011.

[32] V. Korolik, M. R. Alderton, S. C. Smith, J. Chang, and P. J. Coloe, "Isolation and molecular analysis of colonising and noncolonising strains of Campylobacter jejuni and Campylobacter coli following experimental infection of young chickens," Veterinary Microbiology, vol. 60, no. 2-4, pp. 239-249, 1998.

[33] F. M. Colles, N. D. McCarthy, R. Layton, and M. C. J. Maiden, "The prevalence of Campylobacter amongst a free-range broiler breeder flock was primarily affected by flock age," PLoS One, vol. 6, Article ID e22825, 2011.

[34] S. K. Sheppard, F. Colles, J. Richardson et al., "Host association of Campylobacter genotypes transcends geographic variations," Applied and Environmental Microbiology, vol. 76, no. 15, pp. 5269-5277, 2010.

[35] S. Thakur, D. G. White, P. F. McDermott et al., "Genotyping of Campylobacter coli isolated from humans and retail meats using multilocus sequence typing and pulsed-field gel electrophoresis," Journal of Applied Microbiology, vol. 106, no. 5, pp. 17221733, 2009.

[36] P. F. McDermott, S. M. Bodeis, L. L. English et al., "Ciprofloxacin resistance in Campylobacter jejuni evolves rapidly in chickens treated with fluoroquinolones," Journal of Infectious Diseases, vol. 185, no. 6, pp. 837-840, 2002.

[37] N. Luo, O. Sahin, J. Lin, L. O. Michel, and Q. Zhang, "In vivo selection of Campylobacter isolates with high levels of fluoroquinolone resistance associated with gyrA mutations and the function of the CmeABC efflux pump," Antimicrobial Agents and Chemotherapy, vol. 47, no. 1, pp. 390-394, 2003.

[38] S. Cui, B. Ge, J. Zheng, and J. Meng, "Prevalence and antimicrobial resistance of Campylobacter spp. and Salmonella serovars in organic chickens from Maryland retail stores," Applied and Environmental Microbiology, vol. 71, no. 7, pp. 4108-4111, 2005.

[39] J. L. Smith and P. M. Fratamico, "Fluoroquinolone resistance in Campylobacter," Journal of Food Protection, vol. 73, no. 6, pp. 1141-1152, 2010.

[40] K. A. D. M. Oliveira, R. C. S. Mendonca, G. V. De Oliveira, and A. R. Sodre, "Antibiotic resistance of Campylobacter isolated from automated broiler farms," Journal of Food Safety, vol. 26, pp. 82-91, 2005.

[41] X. Chen, G. W. Naren, C. M. Wu et al., "Prevalence and antimicrobial resistance of Campylobacter isolates in broilers from China," Veterinary Microbiology, vol. 144, no. 1-2, pp. 133-139, 2010.

[42] J. M. Kim, J. Hong, W. Bae, C. Koo, S. H. Kim, and Y. H. Park, "Prevalence, antibiograms, and transferable tet(O) plasmid of Campylobacter jejuni and Campylobacter coli isolated from raw chicken, pork, and human clinical cases in Korea," Journal of Food Protection, vol. 73, no. 8, pp. 1430-1437, 2010.

[43] K. Pedersen and A. Wedderkopp, "Resistance to quinolones in Campylobacter jejuni and Campylobacter coli from Danish broilers at farm level," Journal of Applied Microbiology, vol. 94, no. 1, pp. 111-119, 2003.

[44] T. J. Humphrey, F. Jørgensen, J. A. Frost et al., "Prevalence and subtypes of ciprofloxacin-resistant Campylobacter spp. in commercial poultry flocks before, during, and after treatment with fluoroquinolones," Antimicrobial Agents and Chemotherapy, vol. 49, no. 2, pp. 690-698, 2005.

[45] A. Sonnevend, V. O. Rotimi, J. Kolodziejek, A. Usmani, N. Nowotny, and T. Pal, "High level of ciprofloxacin resistance and its molecular background among Campylobacter jejuni strains isolated in the United Arab Emirates," Journal of Medical Microbiology, vol. 55, no. 11, pp. 1533-1538, 2006.

[46] U. Ledergerber, G. Regula, R. Stephan, J. Danuser, B. Bissig, and K. D. C. Stark, "Risk factors for antibiotic resistance in Campylobacter spp. isolated from raw poultry meat in Switzerland," BMC Public Health, vol. 3, article 1, pp. 1-9, 2003.

[47] T. Asai, K. Harada, K. Ishihara et al., "Association of antimicrobial resistance in Campylobacter isolated from food-producing animals with antimicrobial use on farms," Japanese Journal of Infectious Diseases, vol. 60, no. 5, pp. 290-294, 2007.

[48] A. Hernández, M. B. Sánchez, and J. L. Martínez, "Quinolone resistance: much more than predicted," Frontiers in Microbiology, vol. 2, pp. 1-6, 2011.

[49] A. D. Kinana, E. Cardinale, F. Tall et al., "Genetic diversity and quinolone resistance in Campylobacter jejuni isolates from poultry in Senegal," Applied and Environmental Microbiology, vol. 72, no. 5, pp. 3309-3313, 2006.

[50] A. D. Kinana, E. Cardinale, I. Bahsoun et al., "Campylobacter coli isolates derived from chickens in Senegal: diversity, genetic exchange with Campylobacter jejuni and quinolone resistance," Research in Microbiology, vol. 158, no. 2, pp. 138-142, 2007.

[51] I. Habib, W. G. Miller, M. Uyttendaele, K. Houf, and L. De Zutter, "Clonal population structure and antimicrobial resistance of Campylobacter jejuni in chicken meat from Belgium," Applied and Environmental Microbiology, vol. 75, no. 13, pp. 4264-4272, 2009.

[52] W. Gu, R. M. Siletzky, S. Wright, M. Islam, and S. Kathariou, "Antimicrobial susceptibility profiles and strain type diversity of Campylobacter jejuni isolates from turkeys in eastern North Carolina," Applied and Environmental Microbiology, vol. 75, no. 2, pp. 474-482, 2009.

[53] C. B. D’Lima, W. G. Miller, R. E. Mandrell et al., "Clonal population structure and specific genotypes of multidrug-resistant Campylobacter coli from Turkeys," Applied and Environmental Microbiology, vol. 73, no. 7, pp. 2156-2164, 2007.

[54] J. Engberg, J. Neimann, E. M. Nielsen, F. M. Aarestrup, and V. Fussing, "Quinolone-resistant Campylobacter infections in Denmark: risk factors and clinical consequences," Emerging Infectious Diseases, vol. 10, no. 6, pp. 1056-1063, 2004. 
[55] J. Y. M. Johnson, L. M. McMullen, P. Hasselback, M. Louie, G. Jhangri, and L. D. Saunders, "Risk factors for ciprofloxacin resistance in reported Campylobacter infections in southern Alberta," Epidemiology and Infection, vol. 136, no. 7, pp. 903-912, 2008.

[56] M. R. Evans, G. Northey, T. S. Sarvotham, A. L. Hopkins, C. J. Rigby, and D. R. Thomas, "Risk factors for ciprofloxacin-resistant Campylobacter infection in Wales," Journal of Antimicrobial Chemotherapy, vol. 64, no. 2, pp. 424-427, 2009.

[57] S. Kittl, P. Kuhnert, H. Hächler, and B. M. Korczak, "Comparison of genotypes and antibiotic resistance of Campylobacter jejuni isolated from humans and slaughtered chickens in Switzerland," Journal of Applied Microbiology, vol. 110, no. 2, pp. 513-520, 2011.

[58] J. R. C. Ganchingco, K. Kumar, D. M. Stone et al., "Campylobacter coli and C. jejuni isolated from farmed pigs in Grenada, West Indies and their antimicrobial resistance patterns," Journal of Animal Research, vol. 2, no. 3, pp. 237-245, 2012.

[59] K. A. Jolley, M.-S. Chan, and M. C. J. Maiden, "mlstdbNet-distributed multi-locus sequence typing (MLST) databases," BMC Bioinformatics, vol. 5, article 86, 2004. 


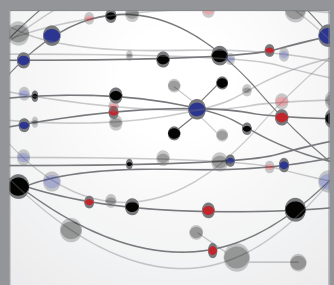

The Scientific World Journal
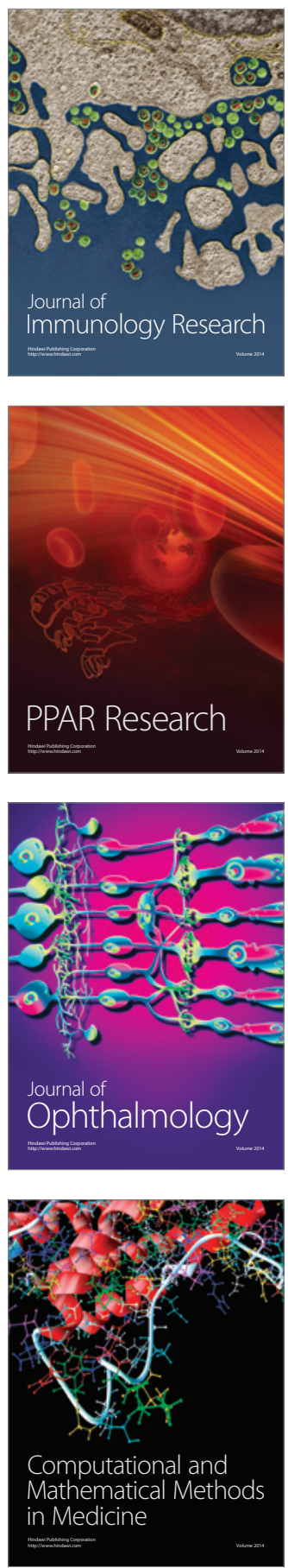

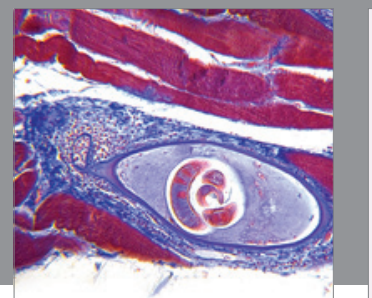

Gastroenterology

Research and Practice
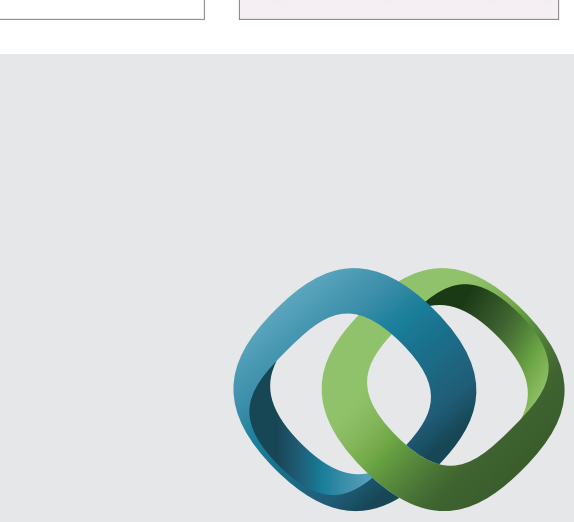

\section{Hindawi}

Submit your manuscripts at

http://www.hindawi.com
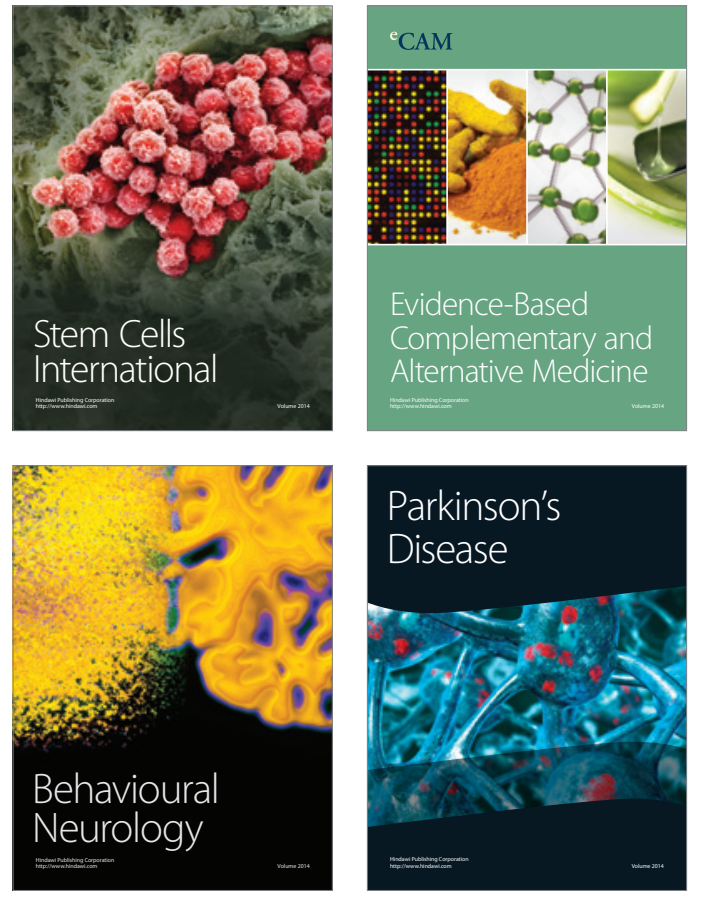
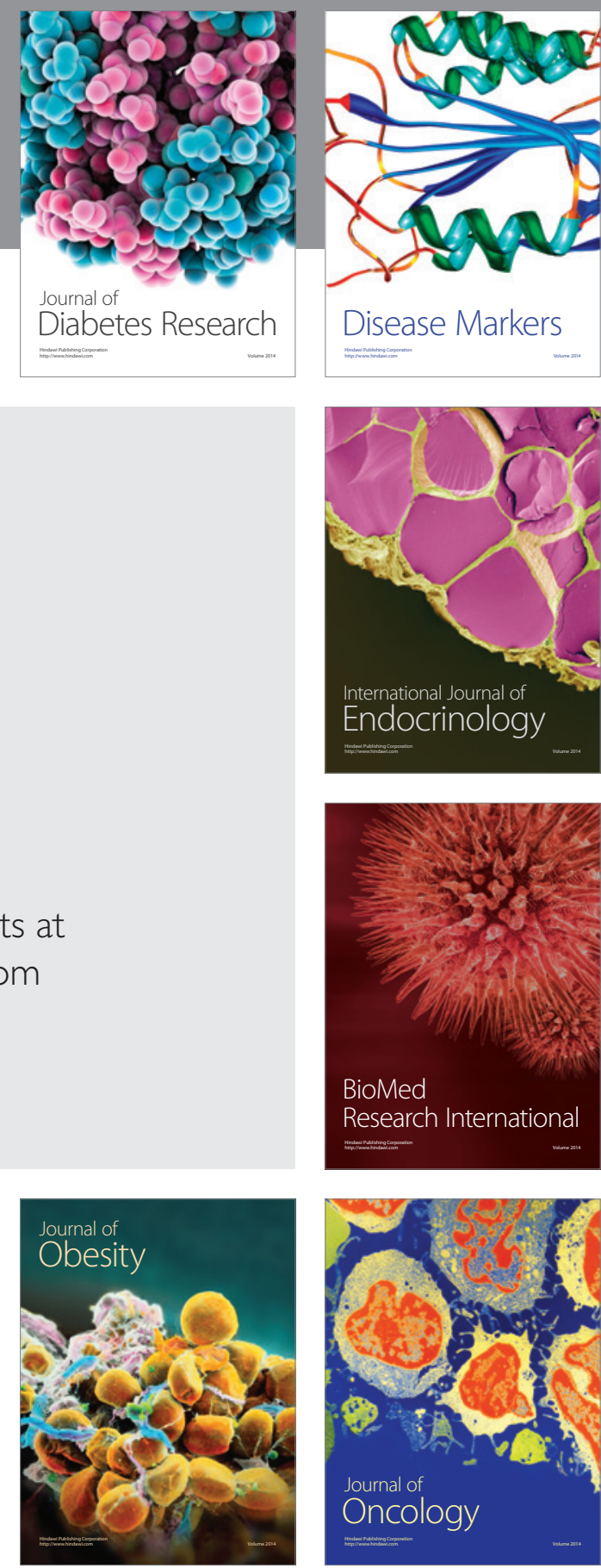

Disease Markers
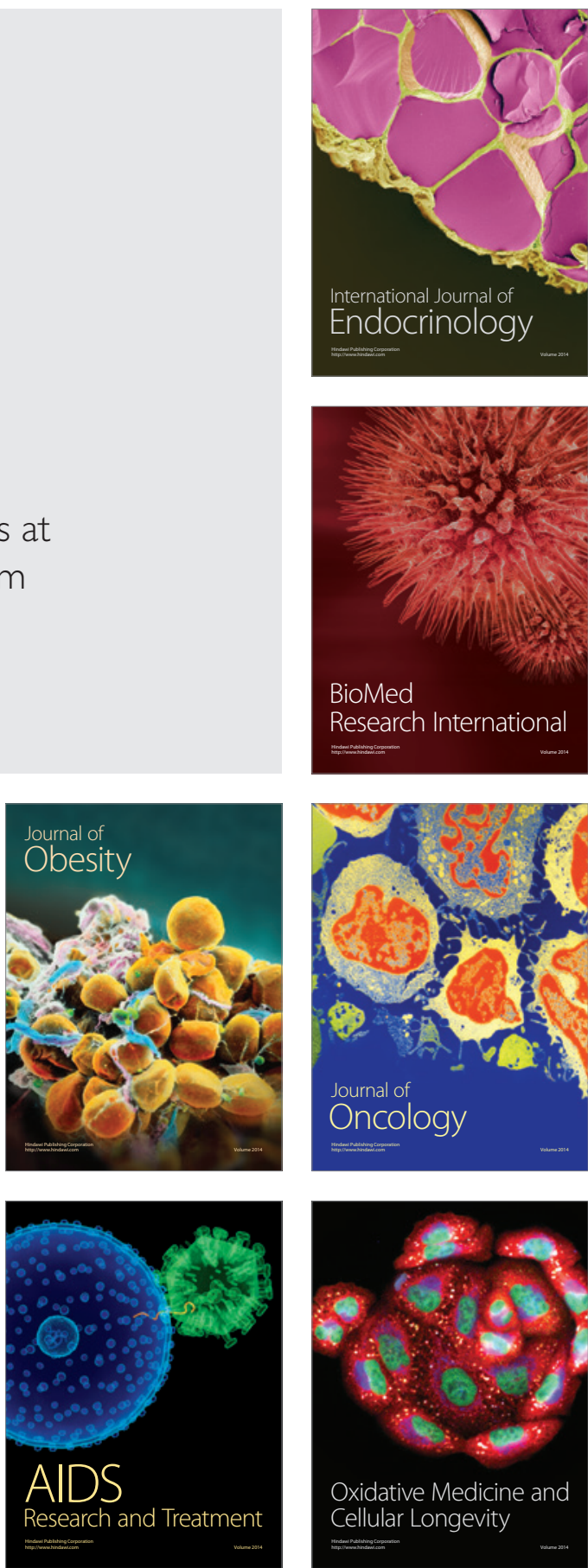